\title{
ZUR SEMANTIK DEUTSCHER ELEMENTE IN TÜRKISCHEN SÄTZEN: Die Mischsprache türkischer Jugendlicher in Mannheim
}

\begin{abstract}
In diesem Beitrag werden die Sprachmischungen, die junge türkischstämmige Migranten in Mannheim/ Deutschland herausgebildet haben, untersucht. Der Fokus liegt auf der Semantik deutscher Elemente in türkischen Strukturen. Die Analyse, die auf den in der Mehrsprachigkeitsforschung eingeführten Kategorien „,kulturelle Entlehnung" und ,spontane Entlehnung" basiert, zeigt, dass nur ein kleiner Teil der Insertionen lexikalische Lücken im Türkischen füllen. Der größte Teil der Insertionen sind spontane Entlehnungen. Sie stammen aus thematischen Bereichen, die das Alltagsleben der jungen Migranten widerspiegeln, und sie werden von Sprechern mit einer hohen Kompetenz in beiden Sprachen verwendet.

This article examines the German-Turkish mixing practices used by young migrants of Turkish origin in Mannheim, Germany. It specially focuses on the semantics of the German elements in Turkish structures. The analysis, based on the categories "cultural borrowings" and "core borrowings", shows that only a small part of the German insertions fill lexical gaps in the Turkish lexicon where no equivalents exist. A large part of the German insertions are ad-hoc borrowings. They derive from thematic areas that reflect situations from the everyday life of the young migrants in Germany. These kinds of insertions are used by Speakers with a high competence in both languages.
\end{abstract}

\section{Einleitung}

In der neueren linguistischen Migrantenforschung stehen die Formen und Funktionen des code-switchings bilingualer Sprechergruppen neben anderen Fragestellungen im Zentrum des Interesses. Eine intensive Forschung hat vor allem der Wechsel innerhalb syntaktischer Konstruktionen ausgelöst. Neben relevanten Ergebnissen haben diese Forschungen auch zur Klärung von Begriffen wie Matrixsprache, Insertion, cultural borrowing und core borrowing geführt. Diese für die vorliegende Untersuchung zentralen Begriffe ebenso wie einige Untersuchungen dazu werden im Folgenden kurz dargestellt. Myers-Scotton bezeichnet die Sprache, die die morphologischen und syntaktischen Regeln liefert, nach denen übernommene Elemente eingebettet werden, als Matrixsprache. ${ }^{1}$ Die in die Matrixsprache morphologisch und syntaktisch eingebetteten Elemente werden im Allgemeinen als Insertionen bezeichnet. ${ }^{2}$ Diese Elemente können sowohl aus einem einzelnen Lexem als auch aus Zusammensetzungen bestehen, die dann als größere lexikalische Einheiten analysiert werden. ${ }^{3}$ Sie dienen dazu, lexikalische Lücken in der Matrixsprache zu füllen. Da diese Insertionen aus der eingebetteten Sprache entlehnt werden, nennt man sie auch Entlehnungen.

Myers-Scotton unterscheidet zwei Klassen von Entlehnungen: cultural borrowing forms (cultural B forms) und core borrowing forms (core B forms). Unter cultural B forms versteht sie Entlehnungen, die Gegenstände und Begriffe repräsentieren, die in der Kultur, zu der die Matrixsprache gehört, neu sind. Somit dienen cultural B forms dazu, eine vorhandene lexikalische Lücke in der Matrixsprache zu füllen. Im Gegensatz zu den cultural B forms besteht bei den core B forms keine lexikalische Notwendigkeit, Entlehnungen aus der eingebetteten Sprache zu verwenden, da für diese in der Matrixsprache sehr

1Zitiert nach Keim (2002, S. 100).

2Vgl. dazu u.a. Backus (1996, S. 94), Keim (2002, S. 107).

3Vgl. dazu u.a. Myers-Scotton (1993, S. 165) und Backus (1996, S. 126 f.). 
nahe und gebräuchliche Äquivalente vorhanden sind. Core B forms stehen somit in Konkurrenz zu äquivalenten Lexemen in der Matrixsprache und sind von ihrem Einsatz her betrachtet von ,grundloser Natur", wie Myers-Scotton das bezeichnet (1993, S. 168172).

Eine im Zusammenhang mit der vorliegenden Untersuchung interessante Arbeit ist die von Backus (1996), der türkisch-niederländische Mischungen, die jugendliche Migrantengruppen in Holland sprechen, unter sematischen und grammatischen Aspekten untersucht. In Bezug auf Insertionen geht er der Frage nach, warum nicht alle Elemente der eingebetteten Sprache die gleiche Chance haben als Insertion gewählt zu werden. Seiner Ansicht nach kann man nur über die Klärung dieser Frage herausfinden, weshalb codeswitching zu Stande kommt und die Form annimmt, die es hat. Nur über die Analyse der semantisch charakteristischen Merkmale der insertierten Elemente können die Motivationen für code-switching aufgedeckt werden (Backus 1996, S. 115). Im Fokus seines Interesses steht die Frage, ob ein insertiertes Element ohne deutliche semantische Motivation gewählt worden ist und es eine semantisch äquivalente Ausdrucksform in der Matrixsprache gibt, oder ob das eingebettete Element keine äquivalente Ausdrucksform in der Matrixsprache hat, also konkurrenzlos ist und der Sprachbenutzer kein in der Matrixsprache etabliertes Lexem verwenden kann. Diese semantische Motivierung einer ,lexikalischen Lücke" bezeichnet er als cultural borrowing. Da er sich in seiner Begrifflichkeit auf die Termini von Myers-Scotton bezieht, bezeichnet er die den cultural borrowings (den kulturspezifischen Entlehnungen) gegenüberstehenden Entlehnungen als core borrowings (1996, S. 118 f.). Interessant ist die Position, die er im Hinblick auf die Beziehung zwischen cultural borrowings und core borrowings vertritt: Sie repräsentieren nicht zwei klar zu unterscheidende Kategorien, sondern core borrowings sind einfach weniger spezifisch als cultural borrowings (1996, S. 121). Begründet wird diese Postion dadurch, dass ein übersetztes Äquivalent oftmals kein wirklich exaktes Äquivalent ist, da das Wissen, das ein Sprecher von einem bestimmten Wort hat, selten den Konnotationen entspricht, die das Äquivalent in der Matrixsprache hat (1996, S. 120). Für core borrowings, also für Entlehnungen ohne klare semantische Motivation, die spontan im Matrixsatz erscheinen, wird im Deutschen auch die Bezeichnung „spontane Entlehnungen" verwendet.

Ein weiteres, von Backus erwähntes Phänomen sind Eigennamen (proper nouns), die er als ,triviale" Beispiele für Einbettungen bezeichnet, da sie so spezifisch sind, dass sie normalerweise nicht vermieden werden können (1996, S. 116 f.). In dieser Darstellung habe ich nur auf die Aspekte von Backus' Untersuchung Bezug genommen, die für die vorliegende Arbeit wichtig sind. Außerdem knüpfe ich an Arbeiten aus dem am Institut für Deutsche Sprache (IDS) durchgeführten, soziolinguistisch-ethnographisch ausgerichten Projekt an, das das Sprach- und Kommunikationsverhalten jugendlicher Migrantengruppen in Mannheim untersucht. Eine der ausgewählten Gruppen ist eine Gruppe junger Frauen, die in der Ingroup vor allem deutsch-türkische Mischungen verwenden. Das Sprach- und Kommunikationsverhalten der Gruppe wird in mehreren Projektpublikationen dargestellt. Diese Arbeiten befassen sich mit soziolingu-

4 Vgl. z.B. Keim (2002, S. 107). 
istischen und stilistischen Aspekten, ${ }^{5}$ beschreiben strukturelle Besonderheiten der sprachlichen Mischungen oder die Türkischkompetenz der Sprecherinnen. ${ }^{6}$

\section{Ziel der Untersuchung, Material und Methode}

In meinem Beitrag werde ich deutsche Insertionen in türkischen Strukturen unter semantischen Aspekten analysieren, und zwar aus einer sprachsystematischen, türkischen Perspektive. Dabei werde ich die insertierten Elemente den Kategorien cultural borrowings (kulturelle Entlehnungen) und core borrowings (spontane Entlehnungen) zuordnen und der Frage nachgehen, inwieweit die Insertionen systematische semantische Lücken füllen oder ad-hoc Entlehnungen sind.

Das Sprachmaterial stammt aus dem am IDS durchgeführten Forschungsprojekt. Es handelt sich um z.T. noch unveröffentlichte Transkripte, die einen Umfang von 920 Seiten haben. Es sind Verschriftlichungen von Tonbanddokumentationen, die während der teilnehmenden Beobachtung bei der Gruppe junger Frauen aufgezeichnet wurden. Die jungen Frauen sind türkischer Herkunft, im Alter zwischen 15 und 22 Jahre und gehören der zweiten Generation türkischer Migranten in Mannheim an. ${ }^{7}$

Die deutschen Insertionen werden zunächst verschiedenen thematischen Bereichen, in denen sie in den Gesprächen auftreten, zugeordnet. ${ }^{8}$ Anschließend wird eine Analyse der deutschen Insertionen vorgenommen, die sie als cultural borrowings (kulturelle Entlehnungen) oder core borrowings (spontane Entlehnungen) klassifiziert. Ausgangspunkt dabei ist die Frage, ob im Türkischen auf der langue-Ebene eine semantisch adäquate Ausdrucksform gefunden werden kann. Auf der Basis der lebensweltlichen Gruppierungen werden dann Angaben zur Frequenz der Wörter sowie zur Verteilung nach Wortarten gemacht. Es werden einzelne Wörter, aber auch mehrgliedrige Kombinationen berücksichtigt, die als lexikalische Einheiten angesehen werden können, wie z.B. zweite Stunde oder ganz normal. Eigennamen wie Städte- und Bezirksnamen oder Namen von Dienstleistungsbetrieben sind ausgeschlossen. Im Rahmen dieser Arbeit werde ich keine Analysen zur morphologischen und syntaktischen Integration des Materials vornehmen. ${ }^{9}$

\section{Analyse und Ergebnisse}

Die in den Transkripten auftretenden deutschen Insertionen umfassen 182 Elemente. Sie wurden in sieben Gruppen eingeteilt. Die erste Gruppe umfasst den Bereich Schule, Ausbildung, Institutionen und Orte, die zweite Gruppe Wörter aus den Lebensbereichen Familie, Freunde, Freizeit und Kommunikation. In der dritten Gruppe werden Beschreibungen, Kommentare, Meinungsäußerungen zusammengefasst. Die vierte Gruppe enthält Wörter zu den Bereichen Kleidung, Körper und Körperpflege und die fünfte Gruppe Elemente aus den Bereichen Einkauf, Essen, Trinken und Feiern. In der sechsten Gruppe

5Vgl. u.a. die Arbeiten von Keim (2001) und Keim/Cindark (2003).

6Vgl. Keim (2002), Cindark/Aslan (2004), Balci (2004) und Sirim (2004).

7Zur Beschreibung dieser Gruppe, den „Powergirls", vgl. Keim in diesem Band.

8Die Zurodnung der deutschen Wörter zu Bereichen, in denen sie in den untersuchten Gesprächen ver wendet wurden, erklärt die Auflistung von Verben wie beobachten, rauskriegen im Bereich „Schule, Ausbildung" und teilnehmen, überlegen im Bereich „Familie, Freunde, Freizeit".

9Vgl. dazu Balci (2004). 
sind Zeitangaben und die siebte Gruppe, die Restgruppe, enthält Elemente, die keiner anderen Gruppe zugeordnet werden konnten.

Im Folgenden werden die einzelnen Gruppen nach Wortarten sortiert dargestellt und die cultural borrowings im Detail beschrieben.

2.1 rste Gruppe: Schule, Ausbildung, Institutionen und Orte

Von den insgesamt 182 Insertionen können 51 Elemente der ersten Gruppe zugeteilt werden, die einen semantischen Bezug zu den Bereichen Schule, Ausbildung, Institutionen und Orte haben. Die 51 Insertionen dieser Gruppe gliedern sich in 37 Substantive und 14 Verben:

Schule, Grundschule, Hauptschule, Real(schule), mittlere Reife, Gymnasium, Probezeit, neunte Klasse, Elternabend, Direktorium, Deutsch, Französisch, Geschichte (Schulfächer), Heft, Arbeitsheft, Thema, Aufsatz, Zeugnis, Mathekurs, Hochquadrat plus, Umkehrung (math. Fachwörter), Stockwerk, Treppe, Computerraum, Computer, Vertrag, Bescheinigung, Arbeitsamt, Anmeldung, (Arzt)Praxis, Date(n), Röntgenbilder, Stadt, Straße, Bahn, Haltestelle, Ampel;

beobachten, erforschen, aufpassen, melden, versauen, einsetzen, einsammeln, verbessern, sitzen bleiben, rechnen, rauskriegen, ausdrucken, eintippen, eingestellt (sein). Als cultural borrowings (kulturelle Entlehnungen), zu denen es keine semantisch adäquaten Ausdrücke im Türkischen gibt, können nur sechs Elemente klassifiziert werden. Fünf davon sind dem deutschen Schulsystem zuzuordnen, das sich strukturell erheblich vom türkischen unterscheidet, d.h. es gibt im Türkischen für viele deutsche Bezeichnungen keine Äquivalente mit derselben semantischen Motivation. In der Türkei gibt es z.B. nach der Grundschule nur eine weiterführende Schulform, die als lise bezeichnet wird; demgegenüber gibt es im deutschen Schulsystem unterschiedliche weiterführende Schulen mit den entsprechenden Bezeichnungen. Im türkischen Schulsystem ist auch das Konzept 'Elternabend' nicht etabliert. Einige Schulen bieten zwar die Möglichkeit mit den Lehrern nach persönlich abgemachten Terminen Kontakt aufzunehmen oder sie organisieren eine Elternversammlung, die als veli toplantisi bezeichnet wird. Der semantische Aspekt, dass der Elternabend abends für berufstätige Eltern durchgeführt wird, fehlt jedoch im türkischen Ausdruck veli toplantisi.

Die als cultural borrowings klassifizierten Elemente kommen in folgenden Äußerungen vor:

14ikisi de mi haupschulede sind

beide auf der Hauptschule

15F. realden șeye düștï ya

$F$. ist doch von der Realschule runter

3. mittlere reifeden bvja von der mittleren Reife auf das BVJ (Berufsvorbereitungsjahr)

4. daha üç sene gymnasiuma. mi gidecem

muss ich etwa noch drei Jahre auf das Gymnasium gehen 


\section{5. çocukların öğretmenlerine elternabend falan ...}

zu den Lehrern der Kinder Elternabend und so ...

Die sechste Entlehnung, die ich als cultural borrowing betrachte, ist die Bezeichnung Stadt in dem Phraseologismus in die Stadt gehen im Sinne von ,im Zentrum der Stadt mit den Einkaufsstraßen, Lokalen und Cafes herumschlendern, bummeln". In der folgenden Äußerung ist das deutsche Lexem Stadt in die türkische Struktur eingebettet, und zwar in der Bedeutung des deutschen Phraseologismus in die Stadt gehen:

6. yarin o zaman stadta. gidek mi sollen wir dann morgen in die Stadt gehen

Im Türkischen würde man den Ort/Platz bzw. den Bezirk nennen, in dem sich Einkaufsmöglichkeiten und Cafes befinden.

Bei den übrigen 45 Insertionen aus dem Bereich „Schule, Ausbildung, Institutionen und Plätze" handelt es sich um spontane Entlehnungen, also um core borrowings. Für sie gäbe es in der türkischen Sprache semantisch adäquate Bezeichnungen.

\subsection{Zweite Gruppe: Familie, Freunde, Freizeit und Kommunikation}

Insgesamt konnten dieser Gruppe 42 Insertionen zugeordnet werden, die zu den Bereichen Familie, Freunde, Freizeit und Kommunikation gehören. Es sind 26 Substantive und 16 Verben:

Generation, Familie, Freund, Mädchentreff, Tschüs, Erwachsene, Mann, Misswahl, Traum, Hektik, Spaß, Horrorfilm, Billardtisch, Ferien, Urlaub, Hotel, Vorschlag, Vorplaner, Tanzkurs, Schwimmbad, Bademeister, Badetuch, Sonnenöl, Telefonzelle, Hörer, Handy;

vertragen, überreden, versprechen, petzen, teilnehmen, gewinnen, träumen, überlegen, besprechen, planen, treffen, grillen, tanzen, erfahren, auflegen, anrufen.

Von 42 Insertionen dieser Gruppe sind nur drei Elemente als cultural borrowings (kulturelle Entlehnungen) zu klassifizieren; d.h. für sie gibt es keine semantisch gleichwertigen Ausdrücke im Türkischen. Bei den ersten beiden handelt es sich um das „öffentliche Schwimmbad":

1. ya da sizinle schwimmbada, gitdiydik oder wo wir mit euch im Schwimmbad waren

2. ya da bu sefer bademeister stres yapmadan önce oder bevor der Bademeister dieses Mal Stress macht

Eine Anlage von der Art Schwimmbad wie es sie in Deutschland gibt, ist in der Türkei nicht öffentlich zugänglich und so gibt es nur die Bezeichnung, die sich auf das Schwimmbecken selbst bezieht: havuz. Auch den Bademeister als Aufsichtsperson in einem Schwimmbad gibt es in der Türkei nicht. Das türkische Wort cankurtaran z.B. bezeichnet die Person, die bei der Lebensrettung am Strand arbeitet; und ein anderes türkisches Wort havuzcu assoziiert eine Person, die für die technische Ausstattung des Schwimmbeckens, für Sauberkeit und Wasserqualität zuständig ist. Beide Ausdrucks- 
möglichkeiten geben nicht den semantischen Inhalt des deutschen Wortes Bademeister wieder.

Der dritte Beleg ist das cultural borrowing Mädchentreff. Es ist die Bezeichnung für eine Freizeitstätte, die es in der Türkei in dieser Art nicht gibt, und dementsprechend bei einer umschreibenden Übersetzung ins Türkische nicht denselben semantischen Gehalt hätte:

3. engeç altida burda olmaliyim mädchentreffde dĕ̌il evde spätestens um sechs muss ich hier sein, nicht im Mädchentreff, zu Hause

Die übrigen 39 Insertionen aus der zweiten Gruppe können als core borrowings, spontane Entlehnungen, klassifiziert werden.

\subsection{Dritte Gruppe: Beschreibungen, Kommentare und Meinungsäußerungen ${ }^{10}$}

In diese Gruppe sind 19 Insertionen eingeordnet worden. Sie alle kommen in Beschreibungen, Kommentaren oder Meinungsäußerungen vor. Von den insgesamt 19 Insertionen sind 11 Adjektive, 5 Verben, 2 Adverbien und 1 Substantiv. In dieser Gruppe sind die meisten Adjektive anzutreffen, da vor allem sie in Beschreibungen verwendet werden. Alle Insertionen dieser Gruppe können als spontane Entlehnungen klassifiziert werden, da der jeweilige semantische Gehalt durch türkische Ausdrücke wiedergegeben werden könnte. Die Elemente dieser Gruppe sind folgende:

voll asozial, ganz eklig, ganz hart, ganz normal, voll, blass, braun, passend, automatisch, kurz, richtig;

angeben, verarschen, nerven, schneller werden, dumm anmachen; überhaupt, irgendwie und Scheiße.

Für Insertionen aus dieser Gruppe gebe ich einige Beispiele:

1. bak suraya vursan bunlar automatisch buraya giriyor schau, wenn du hierhin stößt, gehen die automatisch hier rein

2. zaten nerven yaparsak ablami nerven yapariz wenn wir nerven, nerven wir sowieso meine große Schwester

3. biraz sizin boyunuzda ama erkeğe göre kurz [er] hat etwa eure Größe, aber für einen Mann [zu] kurz

\subsection{Vierte Gruppe: Kleidung, Körper und Körperpflege}

In diese Gruppe, die Elemente zu den Bereichen Kleidung, Körper und Körperpflege enthält, wurden 17 deutsche Elemente eingeordnet, 12 Substantive, 4 Adjektive und ein Verb:

Minirock, Oberteil, Leder, Jungfrau, Titte, Wackelpudding (metaph., weiche Körpermasse), Haare, Föhn, Bauchtraining, Kraft, Kosmetikerin, Bauch, durchsichtig, lesbisch, geil, notgeil und föhnen.

10 Diese Gruppe gehört nicht zu den thematischen Bereichen wie die anderen Gruppen. In dieser Gruppe werden verschiedene Aktivitätstypen zusammengefasst, und die angeführten Insertionen treten in diesen Aktivitätstypen auf. 
Die beiden Ausdrücke geil und notgeil betrachte ich als cultural borrowings (kulturelle Entlehnungen), da sie für jugendsprachliche Formulierungen in Deutschland charakteristisch sind. ${ }^{11}$ Für sie gibt es im Türkischen keine Entsprechungen. Es handelt sich um das qualifizierende Adjektiv geil, das im Sinne von ,toll" oder „wunderbar" verwendet wird, und um das Adjektiv notgeil im Sinne von ,scharf auf Mädchen":

16ne geil olurdu biliyon mu weißt $d u$, wie geil das sein würde

17 o zaten notgeil ya der ist ja sowieso notgeil

Die übrigen 15 Elemente in dieser Gruppe sind spontane Entlehnungen, wie z.B.:

1.böyle durchsichtig biraz so ein bißchen durchsichtig

2.burasi böyle leder gibi

hier ist es so wie Leder

Für die entlehnten deutschen Lexeme gibt es semantisch äquivalente Entsprechungen im Türkischen.

\subsection{Fünfte Gruppe: Einkauf, Essen, Trinken und Feiern}

Den Bereichen Einkauf, Essen, Trinken und Feiern ließen sich insgesamt 16 Insertionen zuordnen, 13 Substantive, ein Verb, ein Adjektiv und ein Adverb: Kasse, Kassenbon, Rückgeld, Eiscafe, Grieche (Restaurant), Flasche, Mineralwasser, Orangensaft, Pfand, Pfandflasche, Sekt, Aschenbecher, Silvester bestellen, besoffen (sein) und ex (trinken). In dieser Gruppe habe ich vier Elemente als cultural borrowings klassifiziert. Zwei Elemente gehören zur Organisation der Flaschenrückgabe in Deutschland:

18bunda var mi pfand ist hier Pfand drauf

19 o zaten pfandflasche das ist sowieso eine Pfandflasche

Die Rückgabe von Getränkeflaschen gegen einen geringen Geldbetrag gibt es in der Türkei nicht; d.h. es gibt für den in den Äußerungen dargestellten Inhalt keine angemessenen Ausdrücke im Türkischen.

Die beiden anderen Elemente bezeichnen Lokalitäten, in denen man essen und trinken kann:

3. eiscafé oturur yer miydin

würdest du dich in ein Eiscafe setzen und essen

$11 \mathrm{Zu}$ jugendsprachlichen Ausdrucksweisen in Deutschland vgl. u.a. Androutsopoulos (1998). 
4. biz seye gittidik ya griecheye wir sind doch dings zum Griechen gegangen

Ähnliche Bezeichnungen im Türkischen geben nicht den semantischen Inhalt der deutschen Wörter wieder. Besonders der Ausdruck zum Griechen (Zugehöriger der Nationalität) gehen als Besuch eines griechischen Restaurants ist semantisch stark motiviert und im Türkischen nicht gebräuchlich. Im Türkischen gibt es die Möglichkeit durch die Bezeichnung des verkauften bzw. hergestellten Gegenstandes und das Suffix -ci, das die Tätigkeit ausführende Person kennzeichnet, die semantische Komponente der Lokalität mitauszudrücken, z.B. dondurmaci, eine Person, die Eis macht oder verkauft und der Ort, wo diese Person Eis verkauft. Aber auch diese Ausdrucksmöglichkeit ist semantisch nicht äquivalent mit einem Wort wie Eiscafe, wenn man berücksichtigt, was in einem Eiscafe alles angeboten wird.

Die übrigen 12 Elemente aus dieser Gruppe können als spontane Entlehnungen klassifiziert werden.

\subsection{Sechste Gruppe: Zeitangaben}

15 deutsche Elemente konnten in diese Gruppe eingeordnet werden, 10 Substantive, 4 Adverbien und eine Präposition:

zweite Stunde, nächste Woche, Montag, Dienstag, Mittwoch, Donnerstag, Freitag, Samstag, drei Uhr, die ganze Zeit, danach, demnächst, auf einmal, heute und bis. In dieser Gruppe gibt es keine kulturspezifischen Entlehnungen, d.h. alle 15 Elemente sind spontane Entlehnungen. Dafür einige Beispiele:

20donnerstag öyleden sonra kapaliymis Donnerstag nachmittags soll [es] geschlossen sein

21drei Uhrda. A. gelecek um drei Uhr kommt A.

3. o da konusmadi die ganze Zeit er hat auch die ganze Zeit nicht gesprochen

4. ben de gülmeye basladim danach böyle ich habe danach auch so angefangen zu lachen

\subsection{Siebte Gruppe: Restgruppe}

Zur Restgruppe gehören 22 deutsche Elemente, die den anderen Gruppen nicht zugeordnet werden konnten, 12 Verben, 4 Substantive, 2 Präpositionen, 2 Adjektive, ein Adverb und eine Konjunktion:

erwarten, drehen, veröffentlichen, auflösen, vertreten, spülen, klopfen, mischen, verletzen, schieben, aufnehmen, mitnehmen, Rotze, Albaner, Lappen, Schublade, mit, aus, zwei, fün, halt, und.

Auch in dieser Gruppe gibt es keine cultural borrowings; alle Insertionen sind spontane Entlehnungen, z.B.: 
22schubladenin anahtari olmasa hiç bi işe yaramiyo wenn der Schlüssel

für die Schublade nicht da ist, bringt es nichts

23 mesela veröffentlichen olacak ya

es wird ja zum Beispiel veröffentlicht

24sen mi spülen yaptin

hast du gespült

25 auflösen olmadi bah ne güzel oldu

es ist nicht aufgelöst, schau wie schön es geworden ist

\section{Tabellarische Zusammenfassung}

Die Ergebnisse der Klassifizierung der 182 deutschen Elemente in verschiedene Lebensbereiche, ihre Zuordnung zu Wortarten und ihre Klassifizierung als cultural borrowings (kulturelle Entlehnungen) oder core borrowings (spontane Entlehnungen) werden im Folgenden tabellarisch und nach ihren prozentualen Anteilen zusammengefasst. Bezogen auf die einzelnen Lebensbereiche ergibt sich folgende Verteilung:

Tabelle 1: Insertionen bezogen auf Lebensbereiche

\begin{tabular}{|l|l|l|l|l|l|l|l|}
\hline Element & $\begin{array}{l}\text { 1. } \\
\text { Gruppe }\end{array}$ & $\begin{array}{l}\text { 2. } \\
\text { Gruppe }\end{array}$ & $\begin{array}{l}\text { 3. } \\
\text { Gruppe }\end{array}$ & $\begin{array}{l}\text { 4. } \\
\text { Gruppe }\end{array}$ & $\begin{array}{l}\mathbf{5} . \\
\text { Gruppe }\end{array}$ & $\begin{array}{l}\text { 6. } \\
\text { Gruppe }\end{array}$ & $\begin{array}{l}\text { Gruppe } \\
\text { Grupp }\end{array}$ \\
\hline $\mathbf{1 8 2}$ & $\mathbf{5 1}$ & $\mathbf{4 2}$ & $\mathbf{1 9}$ & $\mathbf{1 7}$ & $\mathbf{1 6}$ & $\mathbf{1 5}$ & 22 \\
\hline $\mathbf{1 0 0} \%$ & $\mathbf{2 8} \%$ & $\mathbf{2 3} \%$ & $\mathbf{1 0 , 5} \%$ & $\mathbf{9 , 5} \%$ & $\mathbf{9 \%}$ & $8 \%$ & $\mathbf{1 2} \%$ \\
\hline
\end{tabular}

Von insgesamt 182 deutschen Elementen in türkischen Matrixsätzen sind 51 der ersten Gruppe zugeordnet, die die Bereiche „Schule, Ausbildung, Institutionen und Plätze" zusammenfasst. Das macht 28 \% des gesamten Korpus aus. Der hohe Prozentanteil dieser Gruppe lässt sich dadurch erklären, dass die untersuchten Sprecherinnen als Schülerinnen, Studentinnen oder Auszubildende in das Bildungssystem integriert sind und dementsprechend die diesen Lebensbereichen zugehörigen Ausdrücke häufig verwenden. Auch Backus (1996, S. 204) hat in seiner Arbeit über die Mischsprache türkischer Migranten in den Niederlanden festgestellt, dass viele Insertionen aus dem Bereich Bildung und Arbeit kommen.

Die zweite Gruppe umfasst die Bereiche „Familie, Freunde, Freizeit und Kommunikation". Sie beinhaltet 42 Elemente bzw. $23 \%$ des Gesamtinventars. Es verwundert nicht, dass diese Gruppe die zweitgrößte Gruppe ist, wenn man bedenkt, dass die jungen Frauen einer Freizeitgruppe angehören und aktiv am sozialen Leben teilhaben. In der dritten Gruppe sind 10,5 \% der Insertionen enthalten, die im Kontext „Beschreibungen, Kommentare oder Meinungsäußerungen" auftreten; d.h. die Sprecherinnen verwenden in diesen Bereichen überwiegend deutsche Adjektive.

Elemente, die zu den Bereichen „Kleidung, Körper und Körperpflege" gehören, machen 9,5\% aus. Interessant ist, dass im untersuchten Gesprächsmaterial keiner der deutschen Ausdrücke in Verbindung zum Bereich Sexualität steht. Nach den Beobachtungen von 
Backus ist Sexualität die Domäne, in dem seine Informanten Elemente aus dem Niederländischen verwenden (1996, S. 187). D.h. in diesem Bereich verhalten sich die türkischen Mädchen in Mannheim anders. ${ }^{12}$ Es gibt jedoch bei den Insertionen, die zum Bereich „Kleidung" gehören, Ähnlichkeiten zur Untersuchung von Backus (1996, S. 179, 189 f.), der diesen Bereich ebenfalls auflistet. Die fünfte Gruppe, die einen Anteil von 9 \% ausmacht und Wörter aus dem Bereich „Essen, Trinken und Feiern" enthält, zeigt, dass auch in eher auf das häusliche Leben bezogenen Gesprächen deutsche Wörter verwendet werden. Für die jungen Frauen spielt sich dieser Bereich vermutlich auch in deutscher Umgebung ab und ist von bestimmten Gegebenheiten und den dazu gehörenden Wörtern geprägt.

Klassifiziert man die deutschen Insertionen nach Wortarten, entsteht folgende Tabelle:

Tabelle 2: Klassifikation der Insertionen nach Wortarten

\begin{tabular}{|l|l|l|l|l|l|l|}
\hline Element & Subst. & Verben & Adj. & Adv. & Präp & Konj. \\
\hline 182 & 103 & 49 & 18 & 8 & 3 & 1 \\
\hline $100 \%$ & $56,6 \%$ & $26,9 \%$ & $10 \%$ & $4,4 \%$ & $1,6 \%$ & $0,5 \%$ \\
\hline
\end{tabular}

Mit 56,6 \% machen die Substantive die größte Gruppe der im Korpus aufgeführten Wortarten aus. Von 182 Elementen sind das 103. Auch Boeschoten und Verhoeven, die Sprachmischungen bei Migranten der zweiten Generation in den Niederlanden untersuchen, sind in ihrer Analyse der niederländischen Insertionen in türkischen Strukturen zu dem Ergebnis gekommen, dass Substantive die am häufigsten verwendete Wortart ist (Boeschoten/Verhoeven 1985, S. 353). Backus hat in seiner Untersuchung keine Wortarten-Tabelle erstellt, verweist aber darauf (1996, S. 207), dass ähnlich wie in anderen Untersuchungen Substantive an erster Stelle stehen; Auch die übrigen Wortarten sind in seinen Korpora vertreten, allerdings in wesentlich geringeren Anteilen als Substantive. Auch Myers-Scotton (1993, S. 170) weist auf Untersuchungen hin, die ergeben haben, dass unter den Entlehnungen Substantive dominieren.

Fast $27 \%$ der Insertionen sind Verben. Damit nehmen Verben auf der Häufigkeitsskala den zweiten Platz ein. Auch Myers-Scotton berichtet von einem ähnlichen Ergebnis (Myers-Scotton 1993, S. 170). In der Untersuchung von Boeschoten und Verhoeven nehmen Verben zusammen mit Adjektiven den zweiten Platz ein (1985, S. 353). Den dritten Platz nehmen Adjektive mit einem Anteil von $10 \%$ ein, und die übrigen Wortarten sind mit wesentlich kleineren Anteilen vertreten. Das bedeutet, dass 43,4 \% der Entlehnungen nicht-substantivische Elemente sind. Wie der Vergleich mit anderen Untersuchungen ergeben hat, sind im untersuchten Sprachmaterial die Anteile der einzelnen Wortarten an den eingebetteten Elementen ähnlich wie in den Mischstrukturen anderer bilingualer Populationen.

Abschließend werden die Ergebnisse zur Klassifizierung der lexikalischen Elemente als cultural borrowings oder core borrowings tabellarisch präsentiert:

12 Das kann auch mit der Art des ausgesuchten Gesprächsmaterials bzw. der dokumentierten Situtionen zusammenhängen. 
Tabelle 3: Klassifikation der Insertionen als cultrual borrowings und core borrowings

\begin{tabular}{|l|l|l|}
\hline Elemente & cultural borrowings & core borrowings \\
\hline 182 & 15 & 167 \\
\hline $100 \%$ & $8 \%$ & $92 \%$ \\
\hline
\end{tabular}

Von 182 Insertionen konnten nur 15 lexikalische Elemente als cultural borrowings klassifiziert werden. Das sind $8 \%$ des gesamten Materials. Diese Elemente sind semantisch betrachtet wesentlich spezifischer als ihre auf den ersten Blick äquivalent erscheinenden Übersetzungen ins Türkische. Kulturelle Entlehnungen kommen in den Lebensbereichen „Schule, Ausbildung, Institutionen und Orte" (insgesamt sechs Elemente) vor und in den Bereichen „Einkauf, Essen, Trinken und Feiern" (insgesamt vier Elemente). Drei kulturelle Entlehnungen gehören in die Bereiche „Familie, Freunde, Freizeit und Kommunikation" und zwei in die Bereiche „Kleidung, Körper und Körperpflege". Auch wenn Backus in seiner Untersuchung die Insertionen in anderen semantischen Feldern gruppiert, zeigen seine Ergebnisse doch gewisse Übereinstimmungen mit denen der vorliegenden Studie. Backus stellt fest, dass besonders in den Bereichen, die das soziale Leben in Holland wiedergeben wie Kleidung, Verabredungen (Freizeitaktivität) und Sport sowie Bildung, Ausbildung, Arbeit gehäuft cultural borrowings anzutreffen sind (1996, S. 204). Die restlichen 167 lexikalischen Elemente des Korpus sind semantisch nicht spezifisch motiviert und weisen ein Äquivalent in der Matrixsprache auf, so dass für $92 \%$ der deutschen Insertionen eine semantisch äquivalente Ausdrucksmöglichkeit im Türkischen zur Verfügung steht. Eine mögliche Begründung für die Verwendung von core borrowings in diesem Umfang könnte die Feststellung Myers-Scotton sein, nach der bestimmte Arten von Kontaktsituationen den Wunsch fördern, sich mit der Kultur der eingebetteten Sprache zu identifizieren oder zumindest mit Aspekten von ihr. Die Verwendung von core borrowings ist ihrer Meinung nach ein offensichtliches Mittel solcher Identifikation (Myers-Scotton 1993, S. 172). Außerdem hält sie fest, dass core borrowings fast ausschließlich von Sprechern benutzt werden, die sich fließend in beiden Sprachen ausdrücken können (Myers-Scotton 1993, S. 169). Die Feststellung zum Zusammenhang von Kompetenz in beiden Sprachen und hoher Verwendung von core borrowings trifft auch auf die untersuchten Sprecherinnen zu. ${ }^{13}$

\section{Ausblick}

Die vorliegende Untersuchung ging von der Frage aus, für welche der deutschen Ausdrücke, die in türkische Strukturen insertiert wurden, es im Lexikon des Türkischen semantische Äquivalente gibt. Die Zuordnung der deutschen Insertionen zu den Kategorien cultural borrowing oder core borrowing wurde aus einer sprachsystematischen Perspektive vogenommen, von der Ebene der langue aus. Nicht berücksichtigt wurde die Frage, ob es im Gesprächsmaterial der Sprecherinnen für die eingebetteten deutschen Elemente auch türkische Äquivalente gibt. D.h. in der hier vorliegenden Untersuchung

13 Zum Türkischen der jungen Frauen vgl. die Untersuchungen von Cindark/Aslan (2004) und Sirim (2004), die feststellen, dass die Mädchen die türkische Grammatik im Wesentlichen beherrschen. Zum Deutsch vgl. Keim in diesem Band. 
wurde nicht das gesamte Lexikon der Sprecherinnen berücksichtigt, sondern nur die in einer Auswahl von Gesprächstranskripten verwendeten deutschen Insertionen. Aus soziolinguistischer Perspektive ist die Frage interessant, ob im Repertoire der Sprecherinnen auch die äquivalenten türkischen Ausdrücke vorkommen und wenn, in welchen Kontexten sie verwendet werden. Dazu müsste das gesamte Gesprächsmaterial auf türkische Ausdrücke hin durchsucht werden, die zu den deutschen Insertionen äquivalent sind, und auf kontextsprezifische Bedeutungen hin analysiert werden. Es gibt Sprecherinnen, die in einem bestimmten Kontext deutsche und türkische Elemente austauschbar verwenden. ${ }^{14}$ Andere Sprecherinnen haben möglicherweise zu einem deutschen Element nicht das äquivalente türkische Wort und können es auch auf Nachfrage hin nicht produzieren. Das würde auf eine Lücke im türkischen Lexikon hinweisen. Solche Lücken spielen jedoch in der Lebenswelt der Sprecherinnen meist keine Rolle, da das Referenzobjekt durch ein deutsches Lexem bezeichnet werden kann. Das Lexikon solcher Sprecherinnen ist möglicherweise komplementär organisiert. Außerdem kann es sein, dass die zu deutschen Insertionen auf der Systemebene äquivalenten türkischen Ausdrücke für die Sprecherinnen andere Konnotationen haben, in anderen Kontexten verwendet und mit zusätzlichen Bedeutungsaspekten verbunden werden. ${ }^{15}$ Eine kontextbezogene Analyse deutscher und türkischer Elemente könnte Einblick in lebensweltbezogene Bedeutungsveränderungen geben, die sich im Repertoire der Sprecherinnen herausgebildet haben. Soche vergleichenden Analysen könnten Hinweise auf gruppensprachliche Umdeutungen türkischer und deutscher Lexeme geben, was aus soziolinguistischer Perspektive wichtig für die Beschreibung des kommunikativen Stils der Sprecherinnen ist.

\section{Literatur}

Androutsopoulos, Jannis (1998): Deutsche Jugendsprache. Untersuchungen zu ihren Strukturen und Funktionen. Frankfurt/Main: Lang.

Backus, Ad (1996): Two in One. Bilingual Speech of Turkish Immigrants in The Netherlands. Tilburg: Tilburg University Press.

Balci, Yasemin (2004): Das Deutsche im Türkischen oder das Türkische im Deutschen. In: Öztürk, Ilyas (Hg.): Übersetzer- und Übersetzungsprobleme in unserem Zeitalter. Tagungsband des internationalen Übersetzungssymposiums in Sakarya 2004. Sakarya: Tübitak und Sakarya Universität. S. 95-104.

Boeschoten, H./Verhoeven, L. (1985): Integration niederländischer lexikalischer Elemente ins Türkische: Sprachmischung bei Immigranten der ersten und zweiten Generation. In: Linguistische Berichte 98, S. 347-364.

Cindark, Ibrahim/Aslan, Sema (2004): Deutschlandtürkisch? (Manuskript) In: http://www.ids-mannheim.de

Keim, Inken (2001): Die Powergirls. Aspekte des kommunikativen Stils einer Migrantinnengruppe aus Mannheim. In: Jakobs, Eva/Rothkegel, Annely (Hg.): Perspektiven auf Stil. Akten des Kolloquiums zum 60. Geburtstag von Barbara Sandig. Tübingen: Max Niemeyer. S. 375- 400.

Keim, Inken (2002): Sprachvariation und sozialer Stil am Beispiel jugendlicher Migrantinnen türkischer Herkunft in Mannheim. In: Deutsche Sprache. S. 97-123.

14 In einem Gesprächsbeispiel von Keim (in diesem Band, S.) verwendet eines der Mädchen die türkische

Formulierung telefon aç (ruf an) und kurze Zeit später folgt die gemischtsprachliche Form sen anrufen yap (ruf du an).

15 Vgl. z.B. den unterschiedlichen Gebrauch der Nomina Tee und çay oder der Verben lieben etmek und sevmek, den Keim (in diesem Band) bei Kindergartenkindern beschreibt. 
Keim, Inken/Cindark, Ibrahim (2003): Deutsch-türkischer Mischcode in einer Migrantinnengruppe: Form von ,Jugendsprache" oder soziolektales Charakteristikum? In: Neuland, Eva (Hg.): Jugendsprache -Spiegel der Zeit. Tagungsband der internationalen Fachkonferenz in Wuppertal 2001. Frankfurt am Main: Lang. S. 377-393.

Myers-Scotton, Carol (1993): Duelling Languages. Grammatical Structure in Codeswitching. Oxford: Clarendon Press.

Sirim, Emran (2004): Bilinguales Sprachverhalten bei jungen Deutschtürken. Unveröffentlichte Magisterarbeit an der Universität Mannheim.

Dr. Yasemin Balci

Marmara Üniversitesi

Fen-Edebiyat Fakültesi

Alam Dili ve Edebiyati Bölümü

Göztepe Kampüsü

81040 Kadiköy - Istanbul

Türkei

e-mail: balciyasemin@hotmail.com 